\title{
Substrate Crossover Effect and Performance Regeneration of the Biofouled Rotating Air-Cathode in Microbial Fuel Cell
}

OPEN ACCESS

Edited by:

Adrián Escapa,

Universidad de León, Spain

Reviewed by:

Pascal E. Saikaly,

King Abdullah University of Science

and Technology, Saudi Arabia

Feng Zhao,

Institute of Urban Environment (CAS),

China

*Correspondence:

Shuiliang Chen

slchenjxnu@jxnu.edu.cn

Specialty section:

This article was submitted to

Bioenergy and Biofuels,

a section of the journal

Frontiers in Energy Research

Received: 26 June 2018

Accepted: 06 August 2018

Published: 04 September 2018

Citation:

Chen S, Patil SA and Schröder U

(2018) Substrate Crossover Effect and

Performance Regeneration of the

Biofouled Rotating Air-Cathode in

Microbial Fuel Cell.

Front. Energy Res. 6:85.

doi: 10.3389/fenrg.2018.00085

\author{
Shuiliang Chen ${ }^{1,2 *}$, Sunil A. Patil ${ }^{2,3}$ and Uwe Schröder ${ }^{2}$
}

${ }^{1}$ Department of Chemistry and Chemical Engineering, Institute of Advanced Materials, Jiangxi Normal University, Nanchang, China, ${ }^{2}$ Institute of Environmental and Sustainable Chemistry, Technische Universität Braunschweig, Braunschweig, Germany, ${ }^{3}$ Department of Earth and Environmental Sciences, Indian Institute of Science Education and Research, Mohali, India

In case of conventional two-dimensional air-cathodes in microbial fuel cells, biofouling usually covers the catalytic-layer side after a long-term operation and results in performance decrease mainly by obstructing the transfer of $\mathrm{OH}^{-}$ions. This study on a biofouled three-dimensional rotating air-cathode (bio-RAC), demonstrates that besides the $\mathrm{OH}^{-}$effect, substrate crossover acts as a key hindrance to the air-cathode performance. MFC operation and cyclic voltammogram results revealed that about 35\% performance decrease of the bio-RAC performance was caused by the obstruction of oxygen and $\mathrm{OH}^{-}$transfer. It decreased further by 26.8 and $52.7 \%$ in the presence of 3 and $10 \mathrm{mM}$ acetate, respectively, thereby clearly suggesting the impact of substrate crossover on the oxygen reduction reaction at the bio-RAC. In particular, high substrate concentrations exceeded the effect caused by obstruction of oxygen and $\mathrm{OH}^{-}$transfer on the oxygen reduction catalysis. A simple approach of applying a high-speed rotation of about $500 \mathrm{rpm}$ to the biofouled air cathode was proved to be able to recover $85 \%$ of the initial performance of the bio-RAC.

Keywords: microbial fuel cell, biofouled rotating air-cathode, oxygen reduction reaction, substrate crossover, performance regeneration

\section{INTRODUCTION}

Microbial fuel cells (MFCs) belong to emerging microbial electrochemical technologies that utilize the electroactive bacteria to oxidize organic carbon and generate electric power from wastewaters. As one of the key components, the cathode plays a crucial role in determining the performance of MFCs. Although MFCs can adapt to various cathodic reactions by using different electron acceptors (Hamid Rismani-Yazdia et al., 2008; Harnisch and Schroder, 2010), oxygen reduction reaction (ORR) is the most important cathodic reaction for power generation due to its high standard reduction potential $(+0.82 \mathrm{~V}$ vs. SHE) and the abundant availability of oxygen from the air. Efficient electrocatalysts are generally required at the cathode to overcome the effect of sluggish ORR kinetics. Biocatalysts, including bacteria ( $\mathrm{He}$ and Angenent, 2006; Clauwaert et al., 2007) and enzymes (Schaetzle et al., 2009; Higgins et al., 2011) have been reported to catalyze ORR in MFCs, but so far the attainable current density (e.g., by microbial ORR cathodes) and the long term stability (e g., of enzymatic ORR cathodes) do not meet the requirements of a MFC application. Chemical 
ORR catalysts are therefore the material of choice to achieve higher power output and long-term stability. Though a diverse range of materials has been developed for the ORR catalysts (Hamid Rismani-Yazdia et al., 2008; Erable et al., 2012; Liu et al., 2014b), carbon-based materials, such as activated carbon (Zhang et al., 2009, 2014; Dong et al., 2012; Wang et al., 2014), heteroatom-doped carbon (Feng et al., 2011a,b; Chen et al., 2012), especially carbon catalysts derived from sustainable precursors like cellulose (Liu et al., 2014a, 2015b; Yue et al., 2015) and straw (Liu et al., 2015a) are considered as the most feasible ORR catalysts in MFCs. This is primarily due to their good electrocatalytic activity, low-cost and sustainable characteristics.

The most feasible and widely used MFC cathode configuration for wastewater treatment application is the air-cathode, because its use overcomes the limitations associated with the low oxygen solubility in water (Liu and Logan, 2004; Pasupuleti et al., 2015), and thus makes it possible to achieve higher current densities. The popularly used carbon-based ORR catalysts usually possess good biocompatibility but are prone to the colonization by microorganisms and a gradual biofilm formation, which eventually lead to a drastic decrease in its performance. Thick biofilms are usually observed on the air-cathodes after a longperiod operation of MFCs (Liu et al., 2005; Yang et al., 2009; Santoro et al., 2012; Yuan et al., 2013). The biofouling usually covers the catalytic-layer side of the conventional twodimensional air-cathodes (2D-AC), while the oxygen required for the ORR is diffused from the diffusion-layer side to the catalytic sites. Therefore, the performance decrease of the biofouled 2D$\mathrm{AC}$ is mainly because of the obstruction of the $\mathrm{OH}^{-}$transfer and eventual $\mathrm{OH}^{-}$accumulation within the air-cathode (Yuan et al., 2013). Additionally, because the 2D-AC are operated at static conditions, the accumulated $\mathrm{OH}^{-}$ions combine with $\mathrm{Mg}^{2+}$ and $\mathrm{Ca}^{2+}$ ions present in the wastewater and result in the salt deposition within and at the air-cathode, which blocks the diffusion of oxygen to the catalytic sites thereby decreasing its performance further (Oliot et al., 2016).

Recently, we reported a three-dimensional rotating aircathode based on nitrogen and phosphorus co-doped carbon modified graphite brush (GB/NPC-AC) for MFCs and demonstrated that the GB/NPC-AC operated at a slow rotation condition (e.g., $20 \mathrm{rpm}$ ) could generate three times more cathodic current density and double the power output compared to that operated at static conditions (Chen et al., 2018). Similar to the popularly used 2D-AC, the GB/NPC-AC is carbon-based and shows good biocompatibility and thus also gets easily biofouled. The influence of substrate crossover on the ORR performance of abiotic air cathodes has been reported earlier (Harnisch et al., 2009). However, to the best of our knowledge, no such studies have been conducted on the biofouled air-cathodes. In this study, the effect of substrate crossover on the ORR electrocatalytic performance of the rotating biofouled GB/NPC$\mathrm{AC}$ was investigated systematically, and the contributions of the substrate crossover and obstruction of the $\mathrm{OH}^{-}$and oxygen transfer to the performance decrease of this $3 \mathrm{D}$ air-cathode were distinguished. The possibility to recover the ORR performance of the bio-GB/NPC-AC was also investigated by applying rotation at a high speed. For better understanding and consistency, the GB/NPC-AC before and after biofouling and regeneration was denoted as abiotic-GB/NPC-AC, bio-GB/NPC-AC and re-GB/NPC-AC, respectively.

\section{EXPERIMENTAL}

\section{Preparation of the Rotating Air-Cathodes}

Graphite brushes (GBs) with the size of $26 \mathrm{~mm}$ in diameter and $42 \mathrm{~mm}$ in length were self-made using graphite fibers (TORAY) fixed by twisting on two titanium wires. N, P co-doped carbon was coated onto the GB to form the GB/NPC-AC following the method reported in ref (Chen et al., 2018). Typically, the GBs were firstly treated by a $50 \%(\mathrm{w} / \mathrm{w})$ concentrated sulfuric acid to improve their hydrophilicity; then a layer of polyaniline (PANi) was grown onto the GB by a chemical oxidative polymerization process according to the protocol published elsewhere to obtain composite GB/PANi electrode (He et al., 2012). After a de-doping and re-doping process using $0.5 \mathrm{M}$ ammonia and $0.1 \mathrm{M}$ phytic acid solution, respectively, the obtained GB/PANi/PA composite was placed in a high-temperature oven and pyrolyzed at $900{ }^{\circ} \mathrm{C}$ to form the GB/NPC-AC.

\section{Development of the Biofouled Rotating Air-Cathode}

In order to deliberately study biofouling effects of the GB/NPC$\mathrm{AC}$ which unaffected by the anode process, a custom-made, cubic two-chamber MFC was used, as shown in Figures 1A,B. The GB/NPC-AC served as the cathode of the MFC and was operated at a rotation speed of $20 \mathrm{rpm}$ (Chen et al., 2018). GB treated at $450^{\circ} \mathrm{C}$ in the air was used as the anode. An anion exchange membrane (fumasep ${ }^{\circledR}$ ) was used to separate these electrodes. The distance between the two electrodes was $5 \mathrm{~cm}$. Modified M9 medium (50 mM phosphate buffered solution (PBS, $\mathrm{pH}=7.0$ ) containing $20 \mathrm{mM}$ acetate and $12.5 \mathrm{ml} \mathrm{L}^{-1}$ vitamin and $12.5 \mathrm{ml} \mathrm{L}^{-1}$ trace metal solutions) inoculated with $10 \mathrm{~mL}$ real wastewater (Wastewater treatment plant of Steinhof, Braunschweig, Germany) was used as the anolyte. Same medium containing $20 \mathrm{mM}$ acetate was used as catholyte during the biofouling formation of the GB/NPC-AC. The volume of the anolyte and catholyte were 150 and $100 \mathrm{~mL}$, respectively. The two-chambered MFC was operated with acetate only added to the anode chamber initially.

A resistor of $218 \Omega$ was loaded between the anode and cathode electrodes. The cell voltage was recorded every $5 \mathrm{~min}$ by using a multimeter (Keithley 2700). The anode and cathode potentials were also recorded with reference to the $\mathrm{Ag} / \mathrm{AgCl}$ (sat. $\mathrm{KCl},+0.198 \mathrm{~V}$ vs. standard hydrogen electrode) electrode and recorded every $5 \mathrm{~min}$ by a multimeter. Both anolyte and catholyte solutions were refreshed every $48 \mathrm{~h}$. After operating MFCs for about 2 weeks at room temperature $\left(23 \pm 1^{\circ} \mathrm{C}\right)$, the GB/NPC-AC with stable biofouling was obtained.

A single-chambered (separator-free) reactor was also operated as a control. This MFC generated extremely low voltage for about $80 \mathrm{~h}$ (data not shown). Because the anode and cathode shared the same electrolyte, the enhanced oxygen crossover to the anode (due to the rotating air-cathode) most likely hampered the 

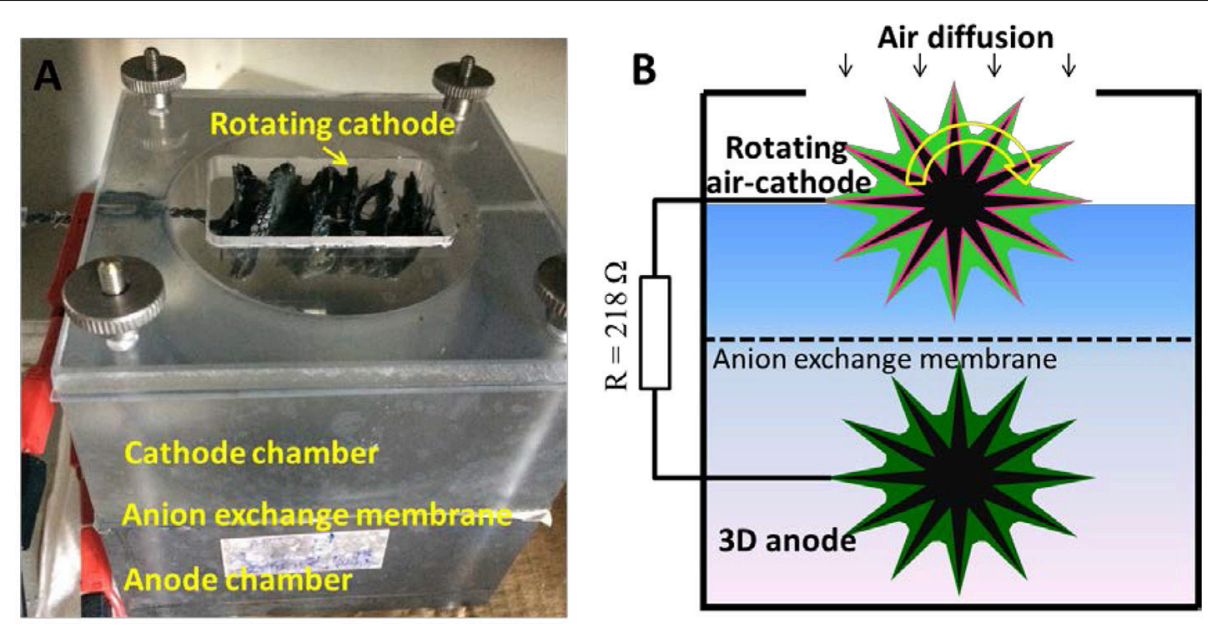

FIGURE 1 | (A) Digital picture and (B) schematic diagram of the MFC set-up used for the development of bio-GB/NPC-AC and subsequent substrate crossover tests.

activity of anaerobic exoelectrogenic bacteria thereby affecting the start-up of this particular MFC.

\section{Substrate Crossover Effect Tests in MFC}

After formation of the bio-GB/NPC-AC, tests to study the effect of substrate crossover on the performance of the MFC and the change in the potential of bio-GB/NPC-AC were conducted. The catholyte was replaced with $100 \mathrm{~mL} 50 \mathrm{mM}$ PBS without acetate, and the anolyte was the same modified M9 medium. The same resistor of $218 \Omega$ was loaded between the bioanode and the bio-GB/NPC-AC. After the initial stable performance, different acetate concentrations, viz., 0.4, 0.8, 1.2, 2.0, and $3.2 \mathrm{mM}$ (from the stock solution of $40 \mathrm{mM}$ ) were then injected successively into the cathode chamber. These acetate concentrations were chosen to cover a representative range of possible substrate crossover concentrations in different types of MFCs (e. g., with and without separators) and according to the literature (Harnisch et al., 2009; Pandit et al., 2012). The cell voltage, anode and cathode potentials under the non-substrate and substrate conditions were recorded.

\section{Electrochemical Tests of Abiotic-GB/NPC-AC and Bio-GB/NPC-AC}

For electrochemical tests, the bio-GB/NPC from the MFC was transferred to a three-electrode system. The bio-GB/NPC served as a working electrode, and an $\mathrm{Ag} / \mathrm{AgCl}$ and a bare $\mathrm{GB}$ were used as the reference and counter electrodes, respectively. Cyclic voltammograms (CVs) of the abiotic-GB/NPC-AC and bioGB/NPC-AC were recorded under the air and $\mathrm{N}_{2}$ atmospheres (potential window of -0.4 to $0.5 \mathrm{~V}$; scan rate of $0.5 \mathrm{mV} \mathrm{s}^{-1}$ ) at a rotation speed of $20 \mathrm{rpm}$. These tests were conducted in the absence and presence of 3 and $10 \mathrm{mM}$ acetate concentrations. Chronoamperometry tests were conducted by applying a constant potential of $-0.3 \mathrm{~V}$ under the similar experimental conditions mentioned above. Acetate concentrations of $0.4,0.8$, 1.2 , and $4.0 \mathrm{mM}$ were added to the cathode chamber, and the current density responses were recorded. These experiments were also conducted in another replicate reactor.

\section{Regeneration of the Bio-GB/NPC-AC}

The regeneration possibility of the bio-GB/NPC-AC was also tested considering the practical applications of such electrodes. The bio-GB/NPC-AC was vertically connected with a mechanical motor and immersed in $50 \mathrm{mM}$ PBS catholyte. The removal of biofouling was tested by bio-GB/NPC-AC at a speed of $500 \mathrm{rpm}$ for $10 \mathrm{~min}$.

\section{RESULTS AND DISCUSSION}

As shown in Figures 2A,B (from 0 to $281 \mathrm{~h}$ ), the dual-chamber MFC equipped with the rotating GB/NPC-AC and with acetate only added to the anode chamber, was able to produce a maximum voltage of approximately $0.461 \mathrm{~V}$ across an external resistance of $218 \Omega$, which corresponded to a current of $2.11 \mathrm{~mA}$.

The main cathodic reaction occurred in the GB/NPC-AC is ORR:

$$
\mathrm{O}_{2}+2 \mathrm{H}_{2} \mathrm{O}+4 e^{-} \rightarrow 4 \mathrm{OH}^{-}
$$

The high voltage generation can be attributed to a highperformance of the rotating GB/NPC-AC, due to the efficient dissolved oxygen diffusion and $\mathrm{OH}^{-}$transport ensured by the rotating condition (Chen et al., 2018). Similar to the popularly used 2D-AC, the carbon-based GB/NPC-AC showed good biocompatibility, and thus was easily colonized by biofilm when exposed to the substrate. Figures 2A,B shows that after refreshment of the catholyte with $50 \mathrm{mM}$ PBS containing $20 \mathrm{mM}$ acetate at the $281 \mathrm{st} \mathrm{h}$, the maximum voltage of the MFC could reach only about $0.422 \mathrm{~V}$. It then rapidly decreased to $0.317 \mathrm{~V}$ and finally recovered to $0.416 \mathrm{~V}$. The voltage further dropped to $0.150 \mathrm{~V}$ when the catholyte was refreshed for the second time after about $24 \mathrm{~h}$, and then recovered to only $0.321 \mathrm{~V}$. It was found that thick biofilms (biofouling) were formed onto the 

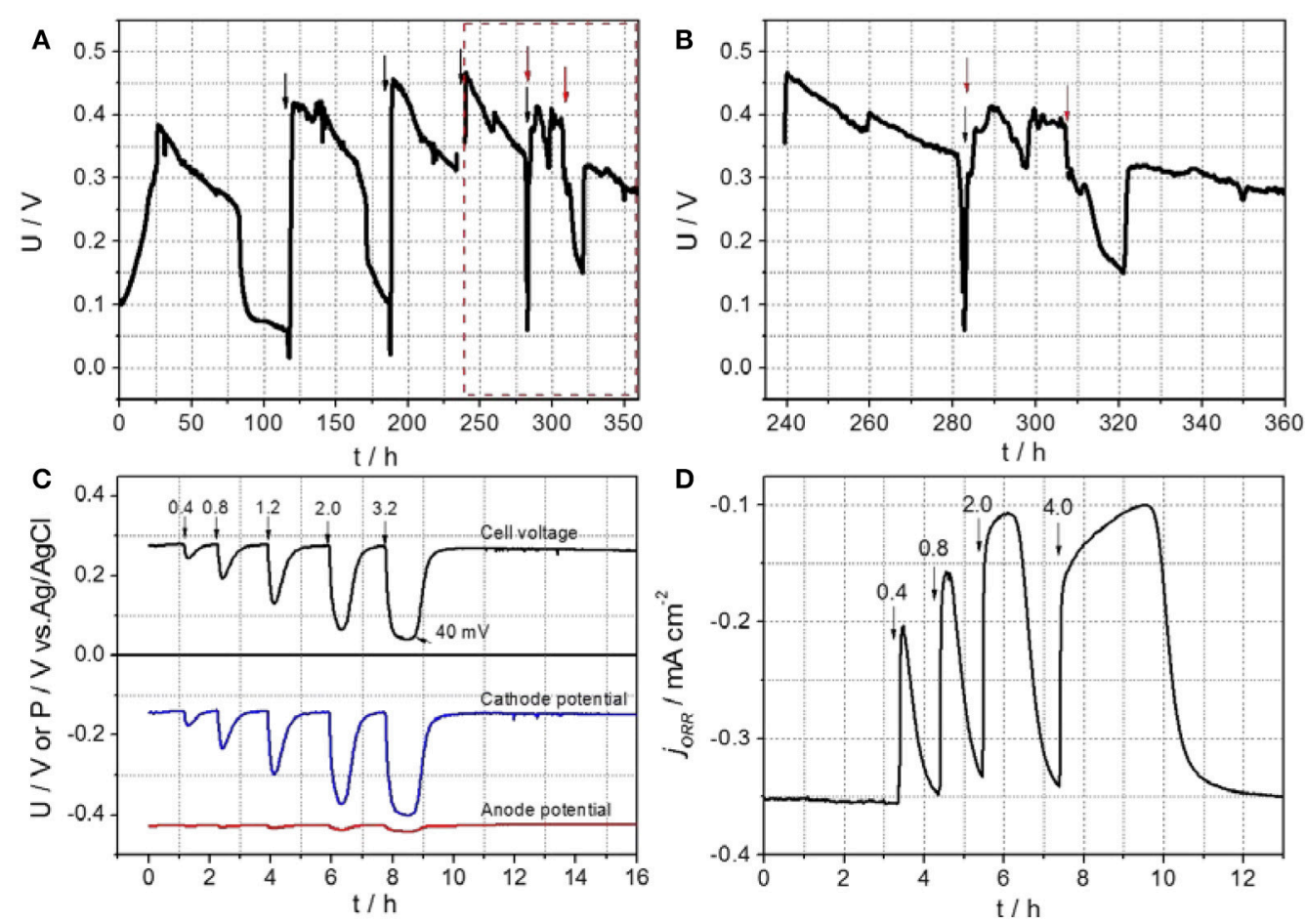

FIGURE 2 | (A,B) Cell voltage curves of the air-cathode MFC during the start-up and biofouling development process; plane (B) is magnified from the dashed plane in (A). The black and red arrows represent the refreshment of anolyte and catholyte solutions, respectively. Substrate crossover effect results of the rotating bio-GB/NPC-AC; (C) profiles of cell voltage along with cathode and anode potentials of the MFC in response to 0.4-3.2 mM acetate additions, and (D) Chronoamperogram recorded in air at a constant potential of $-0.3 \mathrm{~V}$ vs. $\mathrm{Ag} / \mathrm{AgCl}$ (sat. $\mathrm{KCl}$ ). Arrows and numbers indicate injection of acetate solutions with different $\mathrm{mM}$ concentrations.

GB/NPC-AC, which most likely led to the voltage decrease of MFCs.

The substrate crossover effect on the voltage of the MFC was studied with the bio-GB/NPC-AC. As shown in Figure 2C, the MFC generated a stable cell voltage of around $0.280 \mathrm{~V}$ (black curve) across a constant resistance of $218 \Omega$ before injection of acetate solution. The potentials of the bio-GB/NPC-AC and anode were $-0.138 \mathrm{~V}$ (blue curve) and $-0.426 \mathrm{~V}$ (red curve), respectively. After initial injection of acetate to a concentration level of $0.4 \mathrm{mM}$ at the cathode chamber, the cell voltage dropped rapidly from 0.280 to $0.244 \mathrm{~V}$, and then gradually recovered to its initial value. With an increase in the acetate concentrations, the cell voltage decreased further. The cell voltage declined to only approximately $0.040 \mathrm{~V}$ when $3.2 \mathrm{mM}$ acetate was present in the cathode solution. The anode potential showed only a very slight change (Figure 2C, red curve), clearly demonstrating that the decrease of the cell voltage is mainly caused by the decrease in the cathode potential (Figure 2C, blue curve). The complete recovery in the MFC performance after each transient phase of voltage decrease is due to rapid utilization of acetate by the microorganisms through aerobic respiration. The chronoamperogram in Figure 2D confirms the substrate crossover effect of the bio-GB/NPC-AC. After injection of acetate with different concentrations, the ORR current greatly decreased immediately, and then recovered to its initial value of $-0.35 \mathrm{~mA}$ $\mathrm{cm}^{-2}$. The acetate crossover effect in another duplicate MFC measured by CA is shown in Figure 3. It clearly shows the effect of different acetate concentrations (in the range of 0.04 to $2 \mathrm{mM}$ ) on the performance decrease of biofouled air-cathode.

$\mathrm{CV}$ tests on both rotating abiotic-GB/NPC-AC and bioGB/NPC-AC were conducted under $\mathrm{N}_{2}$ atmosphere, air atmosphere, and in the absence and presence of acetate. Figure 4A shows that the CVs of the abiotic-GB/NPC-AC recorded in the presence and absence of acetate are almost identical/overlapped and could generate a cathodic ORR current of up to $-16.74 \mathrm{~mA}$ at the potential of $-0.5 \mathrm{~V}$. It indicates that the abiotic-GB/NPC shows no electrochemical response in the presence of acetate and thus no acetate crossover effect. However, a considerable decrease of ORR current was observed at the bio-GB/NPC-AC. In the absence of acetate, the ORR current of bio-GB/NPC suffers from a great decrease of $34.9 \%$ to $-10.90 \mathrm{~mA}$ (Figure $4 \mathrm{~B}$, black curve). This ORR current decrease can be attributed to the mass transfer blockage by biofouling. The obstruction of oxygen diffusion and $\mathrm{OH}^{-}$ transport by the biofouling at the air-cathode has been reported previously.(Yuan et al., 2013) Refreshing the media with $50 \mathrm{mM}$ PBS containing $3 \mathrm{mM}$ acetate, the ORR current further decreased to $-6.41 \mathrm{~mA}$ (Figure $4 \mathrm{~B}$, red curve), which corresponds to about $26.8 \%$ decrease. With further increase of the acetate concentration to $10 \mathrm{mM}$, a further $25.9 \%$ decrease on the ORR 
current was observed ( $-2.07 \mathrm{~mA}$; Figure 4B, pink curve), which is not far off from the blank value $(-1.12 \mathrm{~mA}$, blue curve in Figure 4A) recorded under $\mathrm{N}_{2}$ atmosphere. The main reason for the ORR current decrease caused by the acetate crossover is the aerobic respiration activity of the biofilm that leads to depletion and eventual blockage of the diffused oxygen to the catalytic sites of the air-cathode. The CVs not only confirmed the substrate crossover effect on the performance of the bioGB/NPC-AC but also distinguished the contributions to the performance decrease from different parameters. These results demonstrate that the substrate crossover aggravates the aircathode performance decrease issue considerably. In particular, at higher concentration (above $5 \mathrm{mM}$ ), the contribution of substrate crossover to the performance decrease of the bioGB/NPC-AC could be higher than that contributed by the obstruction of $\mathrm{OH}^{-}$transfer. For instance, around $52.7 \%$

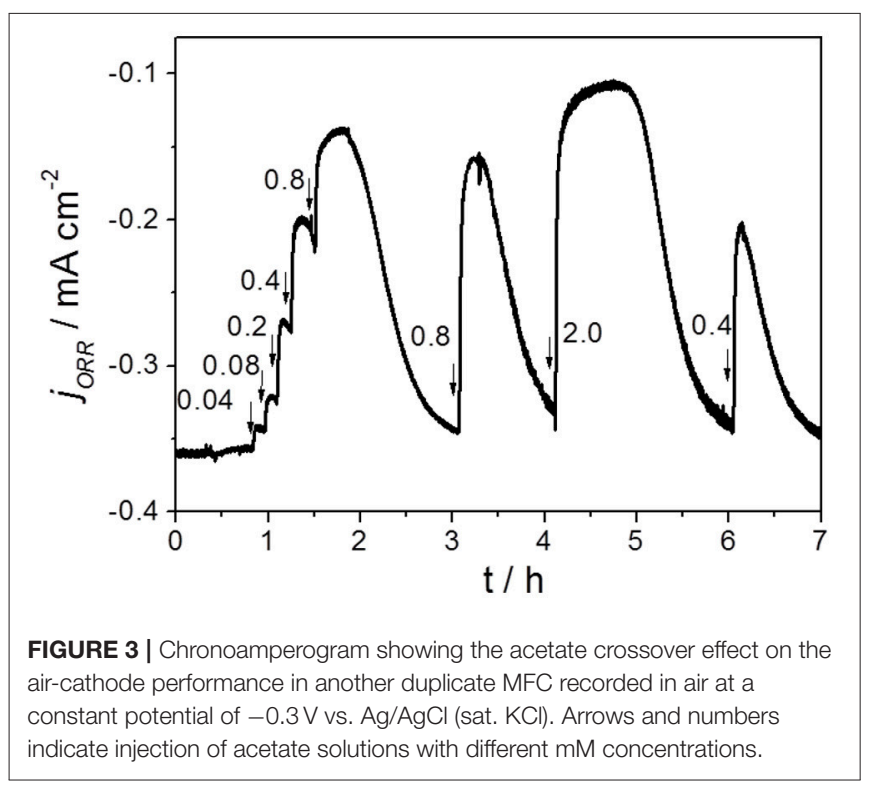

$(26.8+25.9 \%)$ of ORR current decrease of the bio-GB/NPC-AC was caused by $10 \mathrm{mM}$ acetate crossover, even higher than that $(34.9 \%)$ caused by the obstruction of oxygen and $\mathrm{OH}^{-}$ transfer.

The substrate crossover to the cathode is dependent on the type of separator in the MFCs. If an ion exchange membrane is used in the two-chambered air-cathode MFCs, the substrate crossover to the cathode could be in a low level due to the hindrance by the membrane (Pandit et al., 2012; Leong et al., 2013). In such reactor designs, even if some substrate migrates to the cathode chamber, its quick utilization by the biofouled air-cathode would most likely lead to a transient decrease in its performance. However, in the case of porous separator or separator-free MFC designs (Tang et al., 2010; Leong et al., 2013), in which the substrate crossover is more pronounced and occur continuously, the performance of air-cathode would be decreased considerably.

The ORR performance was regenerated by simply applying a high-speed rotation to bio-GB/NPC-AC. As shown in the insets of Figures $4 \mathrm{~A}-\mathrm{C}$, after rotating the bio-GB/NPC-AC at $500 \mathrm{rpm}$ for about $10 \mathrm{~min}$, considerable biofouling was removed from the GB/NPC-AC. The CV recorded at a rotation speed of 20 rpm (Figure 4C, orange curve) revealed that the re-GB/NPCAC produced an ORR current of $-13.75 \mathrm{~mA}$, a recovery of up to $85 \%$ of its initial performance. Moreover, there was no immediate acetate crossover effect on the performance of the GB/NPC after regeneration (Figure 4C, brown curve). This indicates that the recovery was most likely due to the detachment of biomass in this case. If the rotating conditions are applied continuously, then the performance increase would also be due to enhanced oxygen diffusion and $\mathrm{OH}$ - transport as reported elsewhere (Chen et al., 2018). The removal of biofouling through a high-speed rotation was studied only one time with a particular biofouled air-cathode to demonstrate a proof-of concept. It was tested on different biofouled air-cathodes for confirming its reproducibility. However, for its applicability, the long-term effect of this approach needs to be investigated in the future studies.

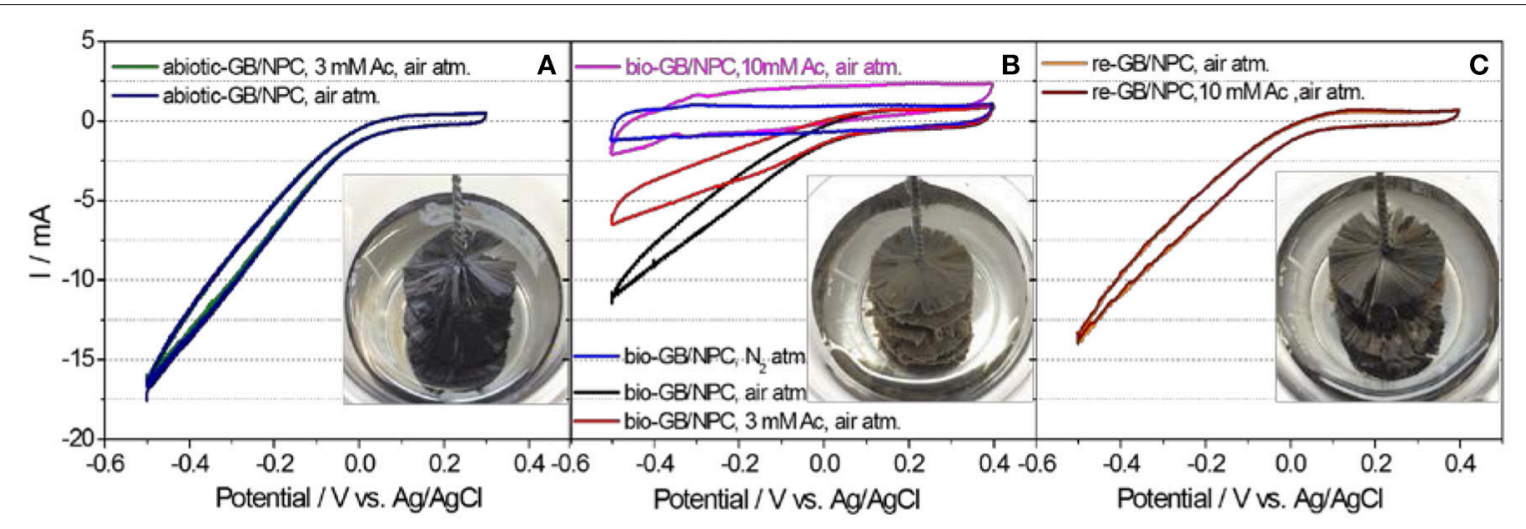

FIGURE 4 | Cyclic voltammograms of rotating (A) abiotic-GB/NPC-AC, (B) bio-GB/NPC-AC, and (C) re-GB/NPC-AC at conditions of air and N2 atmospheres, and in the absence and presence of different acetate concentrations. The scan rate is $0.5 \mathrm{mV} \mathrm{s}{ }^{-1}$. Note: Ac and atm. represent acetate and atmosphere, respectively. Insets of (A-C) are digital photographs of abiotic-GB/NPC-AC, bio-GB/NPC-AC and re-GB/NPC-AC, respectively. 


\section{CONCLUSIONS}

The biofouling at the rotating air-cathode not only played the role of obstructing the transfer of $\mathrm{OH}^{-}$like that in the conventional 2D air-cathode but also blocked the diffusion of oxygen to the catalyst sites mainly due to oxygen depletion through aerobic respiration of the biofilm at the rotating $3 \mathrm{D}$ air-cathode. The contribution of substrate crossover at a high concentration (e.g., over $5 \mathrm{mM}$ ) to the performance decrease of the rotating bio-GB/NPC-AC could be higher than that contributed by the obstruction of oxygen and $\mathrm{OH}^{-}$transfer. Importantly, the bio-GB/NPC-AC could be regenerated by simply removing the biofouling through a high-speed rotation for a short period and recovering up to $85 \%$ of its initial performance. In case of the static air cathode designs, mechanical removal of biofouling or back-flushing of the air-cathodes would be needed. These are time and resource consuming measures. In this context, the facile regeneration characteristic of the rotating air-cathode, as demonstrated in this study, enables it to

\section{REFERENCES}

Chen, S., Chen, Y., He, G., He, S., Schroeder, U., and Hou, H. (2012). Stainless steel mesh supported nitrogen-doped carbon nanofibers for binderfree cathode in microbial fuel cells. Biosens. Bioelectron. 34, 282-285. doi: 10.1016/j.bios.2011.10.049

Chen, S., Patil, S. A., and Schroeder, U. (2018). A high-performance rotating graphite fiber brush air-cathode for microbial fuel cells. Appl Energ. 211, 1089-1094. doi: 10.1016/j.apenergy.2017.12.013

Clauwaert, P., Van der Ha, D., Boon, N., Verbeken, K., Verhaege, M., Rabaey, K., et al. (2007). Open air biocathode enables effective electricity generation with microbial fuel cells. Environ. Sci. Technol. 41, 7564-7569. doi: 10.1021/es0709831

Dong, H., Yu, H., and Wang, X. (2012). Catalysis kinetics and porous analysis of rolling activated carbon-PTFE air-cathode in microbial fuel cells. Environ. Sci. Technol. 46, 13009-13015. doi: 10.1021/es303619a

Erable, B., Féron, D., and Bergel, A. (2012). Microbial catalysis of the oxygen reduction reaction for microbial fuel cells: a review. ChemSusChem 5, 975-987. doi: $10.1002 /$ cssc.201100836

Feng, L., Chen, Y., and Chen, L. (2011a). Easy-to-operate and low-temperature synthesis of gram-scale nitrogen-doped graphene and its application as cathode catalyst in microbial fuel cells. ACS Nano. 5, 9611-9618. doi: 10.1021/nn202906f

Feng, L., Yan, Y., Chen, Y., and Wang, L. (2011b). Nitrogen-doped carbon nanotubes as efficient and durable metal-free cathodic catalysts for oxygen reduction in microbial fuel cells. Energy Environ. Sci. 4, 1892-1899. doi: 10.1039/C1EE01153G

Hamid Rismani-Yazdia, S. M., Carverb, A. D., and Christya, O. H., Tuovinenb, C. (2008). Cathodic limitations in microbial fuel cells: An overview. J. Power Sources 180 683-694. doi: 10.1016/j.jpowsour.2008.02.074

Harnisch, F., and Schroder, U. (2010). From MFC to MXC: chemical and biological cathodes and their potential for microbial bioelectrochemical systems. Chem. Soc. Rev. 39, 4433-4448. doi: 10.1039/c003068f

Harnisch, F., Wirth, S., and Schroeder, U. (2009). Effects of substrate and metabolite crossover on the cathodic oxygen reduction reaction in microbial fuel cells: Platinum vs. iron(II) phthalocyanine based electrodes. Electrochem. Commun. 11, 2253-2256. doi: 10.1016/j.elecom.2009. 10.002

He, S., Hu, X., Chen, S., Hu, H., Hanif, M., and Hou, H. (2012). Needlelike polyaniline nanowires on graphite nanofibers: hierarchical micro/nanoarchitecture for high performance supercapacitors. J. Mater. Chem. 22, 5114-5120. doi: 10.1039/C2JM15668G be considered for practical wastewater treatment application of MFCs.

\section{AUTHOR CONTRIBUTIONS}

SC designed and carried out the experiments, and wrote the manuscript. SP provided constructive suggestions and contributed to the manuscript writing. US supported, supervised the work and contributed to the manuscript writing.

\section{ACKNOWLEDGMENTS}

This work was supported by the National Natural Science Foundation of China (grant 51678281), the Science and Technology Project of Jiangxi Province (grants 20162BCB23024, 20161BCB24005), and the project of Education Department of Jiangxi Province (grants, GJJ150296). SP acknowledges the Alexander von Humboldt Foundation for the financial support.

He, Z., and Angenent, L. T. (2006). Application of bacterial biocathodes in microbial fuel cells. Electroanalysis 18, 2009-2015. doi: 10.1002/elan.2006 03628

Higgins, S. R., Lau, C., Atanassov, P., Minteer, S. D., and Cooney, M. J. (2011). Hybrid biofuel cell: microbial fuel cell with an enzymatic air-breathing cathode. ACS Catalysis 1, 994-997. doi: 10.1021/cs2003142

Leong, J. X., Daud, W. R. W., Ghasemi, M., Ben Liew, K., and Ismail, M. (2013). Ion exchange membranes as separators in microbial fuel cells for bioenergy conversion: a comprehensive review. Renewable Sus. Energy Rev. 28, 575-587. doi: 10.1016/j.rser.2013.08.052

Liu, H., Cheng, S. A., and Logan, B. E. (2005). Power generation in fedbatch microbial fuel cells as a function of ionic strength, temperature, and reactor configuration. Environ. Sci. Technol. 39, 5488-5493. doi: 10.1021/es05 $0316 \mathrm{c}$

Liu, H., and Logan, B. E. (2004). Electricity generation using an air-cathode single chamber microbial fuel cell in the presence and absence of a proton exchange membrane. Environ. Sci. Technol. 38, 4040-4046. doi: 10.1021/es0499344

Liu, L., Xiong, Q., Li, C., Feng, Y., and Chen, S. (2015a). Conversion of straw to nitrogen doped carbon for efficient oxygen reduction catalysts in microbial fuel cells. RSC Advances 5, 89771-89776. doi: 10.1039/C5RA15235F

Liu, Q., Chen, S., Zhou, Y., Zheng, S., Hou, H., and Zhao, F. (2014a). Phosphorus-doped carbon derived from cellulose phosphate as efficient catalyst for air-cathode in microbial fuel cells. J. Power Sources 261, 245-248. doi: 10.1016/j.jpowsour.2014.03.060

Liu, Q., Zhou, Y., Chen, S., Wang, Z., Hou, H., and Zhao, F. (2015b). Cellulosederived nitrogen and phosphorus dual-doped carbon as high performance oxygen reduction catalyst in microbial fuel cell. J. Power Sources 273, 1189-1193. doi: 10.1016/j.jpowsour.2014.09.102

Liu, X. W., Li, W. W., and Yu, H. Q. (2014b). Cathodic catalysts in bioelectrochemical systems for energy recovery from wastewater. Chem. Soc. Rev. 43, 7718-7745. doi: 10.1039/C3CS60130G

Oliot, M., Galier, S., de Balmann, H. R., and Bergel, A. (2016). Ion transport in microbial fuel cells: Key roles, theory and critical review. Appl. Energ. 183, 1682-1704. doi: 10.1016/j.apenergy.2016.09.043

Pandit, S., Ghosh, S., Ghangrekar, M. M., and Das, D. (2012). Performance of an anion exchange membrane in association with cathodic parameters in a dual chamber microbial fuel cell. Int. J. Hydrogen Energy 37, 9383-9392. doi: 10.1016/j.ijhydene.2012.03.011

Pasupuleti, S. B., Srikanth, S., Venkata Mohan, S., and Pant, D. (2015). Continuous mode operation of microbial fuel cell (MFC) stack with dual gas diffusion cathode design for the treatment of dark fermentation effluent. Int. J. Hydrogen Energy 40, 12424-12435. doi: 10.1016/j.ijhydene.2015.07.049 
Santoro, C., Lei, Y., Li, B., and Cristiani, P. (2012). Power generation from wastewater using single chamber microbial fuel cells (MFCs) with platinum-free cathodes and pre-colonized anodes. Biochem. Eng. J. 62, 8-16. doi: 10.1016/j.bej.2011.12.006

Schaetzle, O., Barriere, F., and Schroeder, U. (2009). An improved microbial fuel cell with laccase as the oxygen reduction catalyst. Energy Environ. Sci. 2, 96-99. doi: $10.1039 / \mathrm{b} 815331 \mathrm{k}$

Tang, X., Guo, K., Li, H., Du, Z., and Tian, J. (2010). Microfiltration membrane performance in two-chamber microbial fuel cells. Biochem. Eng. J. 52, 194-198. doi: 10.1016/j.bej.2010.08.007

Wang, X., Feng, C., Ding, N., Zhang, Q., Li, N., Li, X., et al. (2014). Accelerated $\mathrm{OH}$ - transport in activated carbon air cathode by modification of quaternary ammonium for microbial fuel cells. Environ. Sci. Technol. 48, 4191-4198. doi: 10.1021/es5002506

Yang, S., Jia, B., and Liu, H. (2009). Effects of the Pt loading side and cathode-biofilm on the performance of a membrane-less and singlechamber microbial fuel cell. Bioresour. Technol. 100, 1197-1202. doi: 10.1016/j.biortech.2008.08.005

Yuan, Y., Zhou, S., and Tang, J. (2013). In situ investigation of cathode and local biofilm microenvironments reveals important roles of $\mathrm{OH}$ - and oxygen transport in microbial fuel cells. Environ. Sci. Technol. 47, 4911-4917. doi: $10.1021 /$ es400045s
Yue, G., Meng, K., and Liu, Q. (2015). One-step synthesis of N-doped carbon and its application as a cost-efficient catalyst for the oxygen reduction reaction in microbial fuel cells. ChemPlusChem 80, 1133-1138. doi: 10.1002/cplu.201500057

Zhang, F., Cheng, S., Pant, D., Van Bogaert, G., and Logan, B. E. (2009). Power generation using an activated carbon and metal mesh cathode in a microbial fuel cell. Electrochem. Commun. 11, 2177-2179. doi: 10.1016/j.elecom.2009.09.024

Zhang, X., Xia, X., Ivanov, I., Huang, X., and Logan, B. E. (2014). Enhanced activated carbon cathode performance for microbial fuel cell by blending carbon black. Environ. Sci. Technol. 48, 2075-2081. doi: 10.1021/es405029y

Conflict of Interest Statement: The authors declare that the research was conducted in the absence of any commercial or financial relationships that could be construed as a potential conflict of interest.

Copyright $(2018$ Chen, Patil and Schröder. This is an open-access article distributed under the terms of the Creative Commons Attribution License (CC BY). The use, distribution or reproduction in other forums is permitted, provided the original author(s) and the copyright owner(s) are credited and that the original publication in this journal is cited, in accordance with accepted academic practice. No use, distribution or reproduction is permitted which does not comply with these terms. 